\title{
Proceso de fundición en molde de arena: diseño, simulación e impresión 3D
}

\author{
Sand Mold Casting Process: Design, Simulation and 3D Print
}

Carlos Borja-Soto ${ }^{a}$, Justo Montiel-Hernández ${ }^{b}$, Víctor Jiménez de la Paz ${ }^{c}$, David Luqueño de la Rosa ${ }^{d}$

\begin{abstract}
:
This article contains the results obtained from the simulation of the sand mold casting process for A 380 aluminium alloy. The Young's modulus values of the A 380 alloy as a function of temperature were also included. The use of computer packages represents advantages in the design of mechanical parts. Finally, the results of 3D printing in obtaining a scale-size model are shown.
\end{abstract}

Keywords:

Sand mold, simulation, mechanical properties, solidification, alloys, phase diagram

\section{Resumen:}

Este artículo contiene los resultados obtenidos a partir de la simulación del proceso de fundición en molde de arena para una aleación de aluminio A 380. También fueron incluidos los valores del módulo de Young de la aleación A 380 en función de la temperatura. El uso de los paquetes informáticos representa ventajas en el diseño de piezas mecánicas. Finalmente, son mostrados los resultad os de la impresión 3D en la obtención de un modelo a tamaño escala.

\section{Palabras Clave:}

Molde de arena, simulación, propiedades mecánicas, solidificación, aleaciones, diagrama de fases

\section{Introducción}

El uso de los metales y sus aleaciones en aplicaciones de ingeniería es muy amplio. Las aplicaciones de estos materiales pueden ser funcionales y estructurales, así como lo es para otros tipos de materiales. En el caso de las aplicaciones estructurales, los materiales estarán bajo condiciones de esfuerzos mecánicos principalmente. Por otra parte, cuando las aplicaciones son del tipo funcional, las propiedades que principalmente son promovidas en el diseño son diferentes a las propiedades mecánicas, por ejemplo, resistividad eléctrica, conductividad eléctrica, magnetización de saturación, entre muchas otras. (Smith, 2007), (Groover, 2007)
El diseño de piezas mecánicas metálicas requiere determinar las propiedades mecánicas de las piezas que pueden ser obtenidas a partir de uno o varios procesos de manufactura. Es decir, los materiales y los procesos de manufactura involucrados en la obtención de dichas piezas. Las aleaciones metálicas puedes ser clasificadas en ferrosas y no ferrosas. En el caso de las aleaciones ferrosas, el elemento metálico que se encuentra en la composición química en mayor cantidad es Fe, tales como son los aceros. En las aleaciones no ferrosas, las aleaciones tienen en mayor cantidad otro elemento metálico diferente al $\mathrm{Fe}$, tal es el caso de las aleaciones base Al, Cu, Ti, Zr, entre otros. (Smith, 2007)

\footnotetext{
Autor de Correspondencia, Universidad Autónoma del Estado de Hidalgo, https://orcid.org/0000-0003-3385-8348, Email: carlos_borja@uaeh.edu.mx

b Universidad Autónoma del Estado de Hidalgo, https://orcid.org/0000-0001-6890-6069, Email: justo_montiel@uaeh.edu.mx

c Universidad Autónoma del Estado de Hidalgo, https://orcid.org//0000-0001-5661-6339, Email: jimenezdelapazvictoralonso9@ gmail.com

d Universidad Autónoma del Estado de Hidalgo, https://orcid.org/0000-0001-5413-8089, Email: dawik23ex@ gmail.com
} 
El proceso de fundición es muy usado para la obtención de piezas de aplicación funcional y estructural. El proceso consiste en elevar la temperatura del material a procesar en un horno, el cual puede usar como medio de calentamiento la energía eléctrica, energía magnética e inclusive aquellos que aprovechan la liberación de calor del proceso de combustión.

Al elevar la temperatura de la carga metálica en el homo es posible llevarla hasta por encima de temperatura de fusión, con la finalidad de que ocu rra la tran sformación del estado sólido al líquido. Cuando el material se encuentra en estado líquido y tiene la composición química requerida; es colocado den tro de un molde. La cavidad del molde determinará la forma geométrica de las piezas obtenidas.

Existen varios tipos de moldes, estos pueden ser clasificados en moldes permanentes y desechables. La diferencia entre estos, es que los primeros pueden ser reutilizables.

En el proceso de fundición en moldes de arena, los moldes son obtenidos por la compactación de arena sílica, la cual es previamente mezclada con un aglutinante. La cavidad del molde es generada por una pieza denominada "modelo" que es previamente diseñada y tiene dimensiones aproximadas a la pieza que se desea obtener a partir del molde.

El modelo debe ser diseñado tomando en cuenta factores tales como la contracción volumétrica del material, ángulos en la superficie (ángulos de salida) que permitan una fácil extracción del modelo y evitar que parte de la arena se derrumbe.

En la extracción del modelo es preciso tener los cuidados necesarios para evitar que existan partículas de arena en cavidad interior del molde y en caso de haberlas es importante retirarlas. La presencia de partículas de arena en el interior del molde debe ser evitada antes de colar el líquido al molde para evitar defectos superficiales en las piezas e inclusive queden atrapadas en el material como inclusiones no metálicas. El proceso de fundición en moldes de arena, así como muchos otros han sido ampliamente descritos en la literatura. (Groover, 2007) (Smith, 2007)

La figura 1 muestra esquemáticamente que en el diseño de un proceso de fundición en moldes de arena deben ser considerados principalmente los siguientes aspectos:

- Características del molde.

- Colada del líquido.

\section{- Propiedades de los materiales a procesar.}

En la actualidad es posible el uso de programas informáticos que permiten predecir en forma aproximada el comportamiento de los fluidos dentro del molde, los cuales son una herramienta que permiten incrementar las probabilidades de obtener piezas que cumplan con las características geométricas diseñadas y propiedades físicas, entre otras. Existen paquetes informáticos que permiten determinar rápidamente las diferentes propiedades de los metales o aleaciones cuando estos solidifican a determinadas velocidades de enfriamiento. Entre las propiedades que pueden ser estimadas por medio de programas informáticos se encuentran el módulo de elasticidad, dureza, densidad, conductividad eléctrica, resistividad eléctrica, conductividad térmica, entre otras.

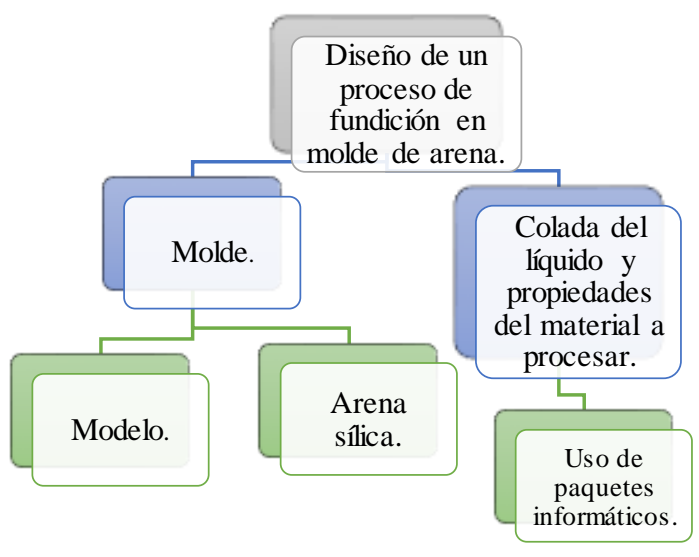

Figura 1. Esquema general del diseño de un proceso de fundición en moldes de arena.

\section{Metodología}

En el diseño de una pieza de la aleación de alu minio A 380 fueron usados los paquetes informáticos solidworks, jmat y ultimaker cura para llevar a cabo la simulación del proceso de fundición en molde de arena, diseño de modelo, la determinación del módulo de Young de la aleación y la obtención de un modelo a escala por medio de impresión 3D.

Inicialmente, fueron determinadas las características geométricas del modelo. Posteriormente, fueron generados los archivos electrónicos para la impresión del modelo y la simulación del proceso de fundición. 
La impresión en 3D fue llevada a cabo en una impresora de control numérico 3D Creality. El espesor por capa aplicada fue de $0.2 \mathrm{~mm}$. El modelo fue elaborado con polímero PLA. La pieza fue impresa a una escala de 1:75.

El archivo electrónico con extensión iges fue usado para llevar a cabo el proceso de simulación del llenado del molde. El material considerado en el diseño y simulación del proceso de fundición fue aleación de aluminio A 380 . 3.Los valores del módulo de Young en función de la temperatura para la aleación A 380, fueron determinados a partir de un programa informático especializado del área metalúrgica jmat.

\section{Resultados}

Las figuras $2-3$ muestran el modelo generado por medio del programa solidworks. La pieza mostrada podría ser usada en la conformación de las tapas laterales de rodillos. Sin embargo, la variedad de piezas o dispositivos mecánicos que pueden ser diseñados con solidworks es enorme.

En el diseño del modelo fueron considerados los ángulos de salida para facilitar la extracción del modelo para la obtención de la cavidad interior del molde de arena. Las protuberancias cilíndricas que sobresalen perpendicularmente de la cara de la pieza mostrada en la figura 2, tienen la función de ser un reservorio de material para evitar los defectos volumétricos y superficiales en la pieza final debido a la contracción originada por la disminución de la temperatura durante la solidificación. Las figuras 2 a 4 muestran un volumen en forma de "L" que se encuentra en la zona periférica de la pieza, por medio de ese volumen se lleva a cabo la alimentación del material líquido al molde.

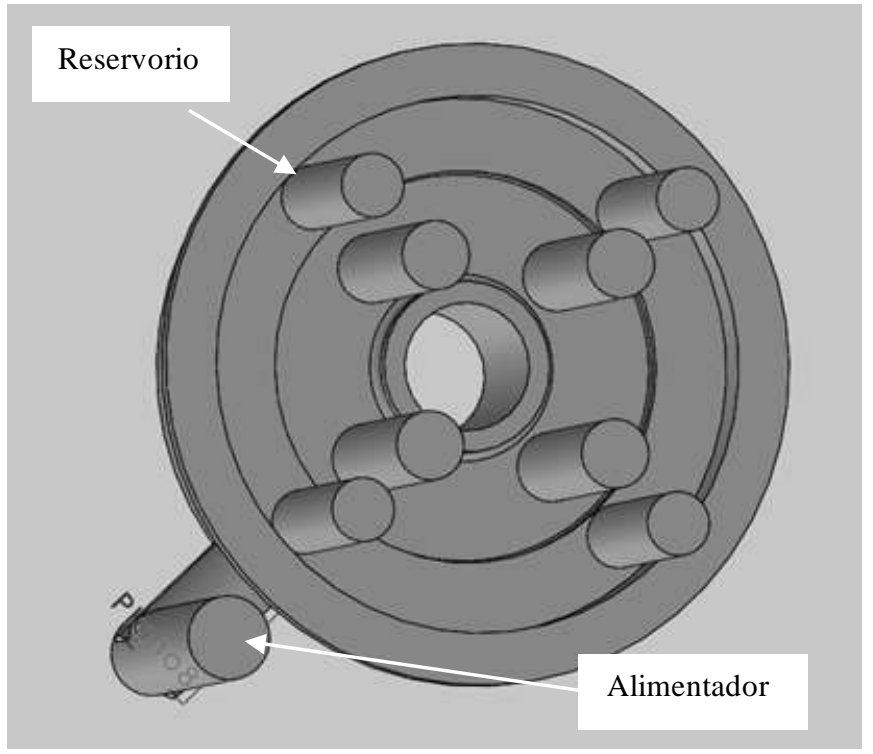

Figura 2. Vista superior.

Después de la solidificación del material es necesario que las protuberancias que sobresalen de la pieza de interés sean removidas por medio de herramientas de corte del tipo abrasivo. La pieza obtenida por medio de la fundición en molde de arena tendría que ser maquinada por medio de un torno y un cepillo de codo. El maquinado de las partes cilíndricas de la pieza tendría que ser llevado a cabo en un torno, mientas que el maquinado del cuñero interior de la pieza sería tallado en un cepillo de codo. Las variables o parámetros de maquinado tendrían que ser determinados adecuadamente, de acuerdo a tipo de material, dimensiones de la pieza, herramienta de corte, entre otros.

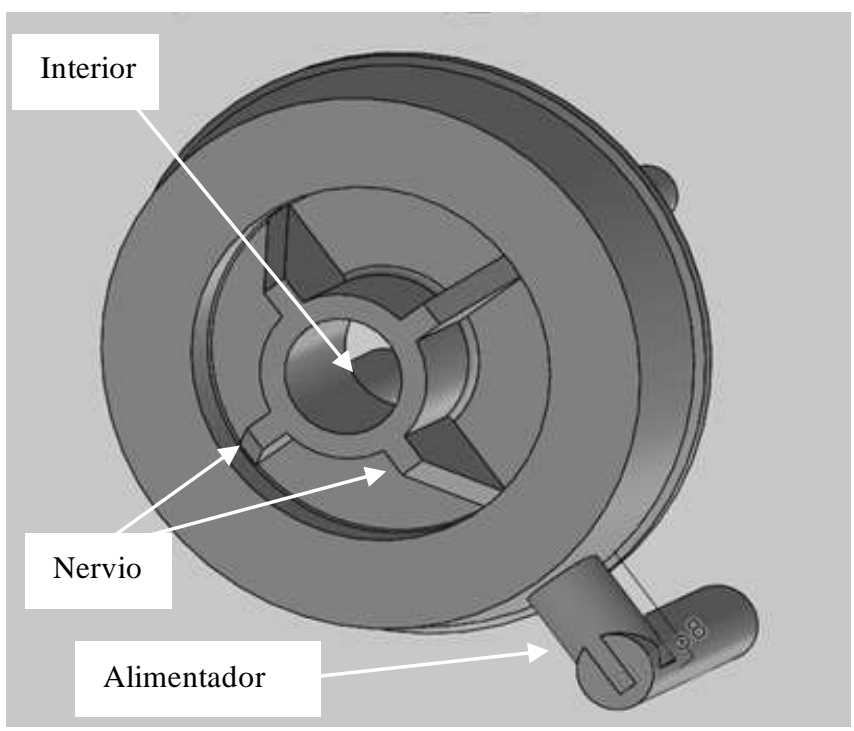

Figura 3. Vista inferior. Diámetro interior de la pieza, nervios de refuerzo y alimentador 
La figura 4 muestra el resultado de la simulación del llenado de la cavidad del molde por la aleación A 380 en estado líquido a una temperatura de $700^{\circ} \mathrm{C}$.

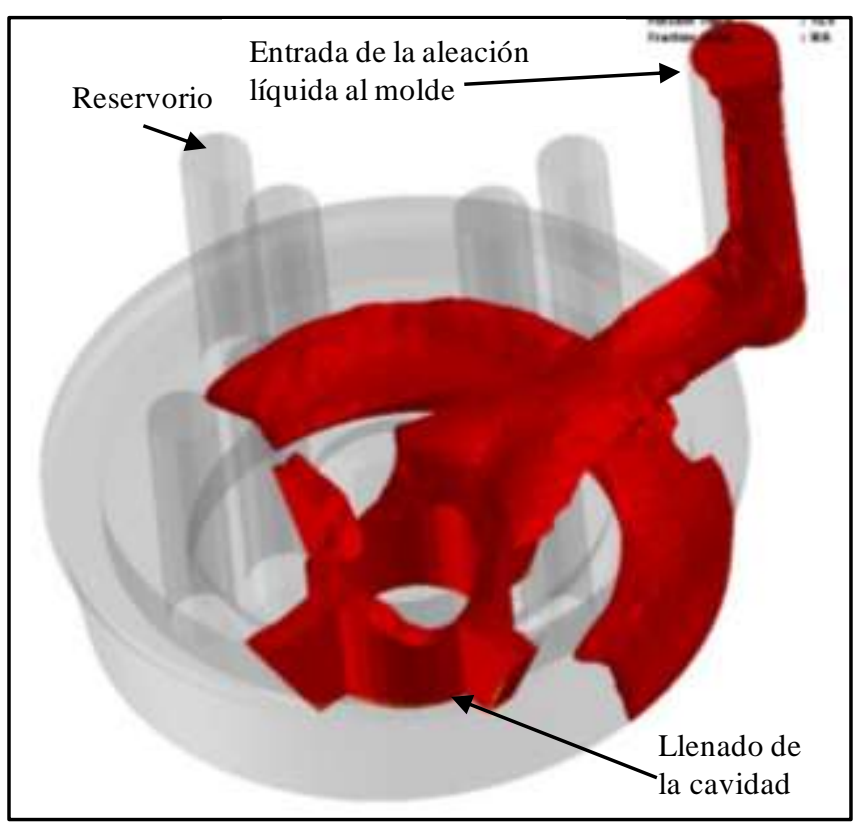

Figura 4. Inicio del llenado de la cavidad del molde de arena.

En la simulación del proceso del llenado del molde de arena son considerados principalmente los siguientes factores:

- Colado del material en molde de arena.

- Temperatura de la aleación al ser colada al molde.

- Transferencia de calor.

- Enfriamiento al aire.

- Temperatura del molde a $25^{\circ} \mathrm{C}$ antes de interaccionar con la aleación líquida.

- Llenado por gravedad.

- Temperatura final de la aleación hasta los $300^{\circ} \mathrm{C}$.

La figura 5 muestra los gradientes de temperatura en la pieza durante la solidificación. Las partes más delgadas de la pieza son las primeras en solidificar, de acuerdo a los gradientes de temperatu ra que pueden identificarse en dicha figura. De acuerdo a los resultados es posible la obtención de la pieza por medio de fundición en molde de arena, dado que no fueron identificados defectos volumétricos o falta de llenado del molde. Sin embargo, modelo de fundición puede ser mejorado cambiando la posición de los reservorios, cambiando la posición del alimentador y las dimensiones de estos.

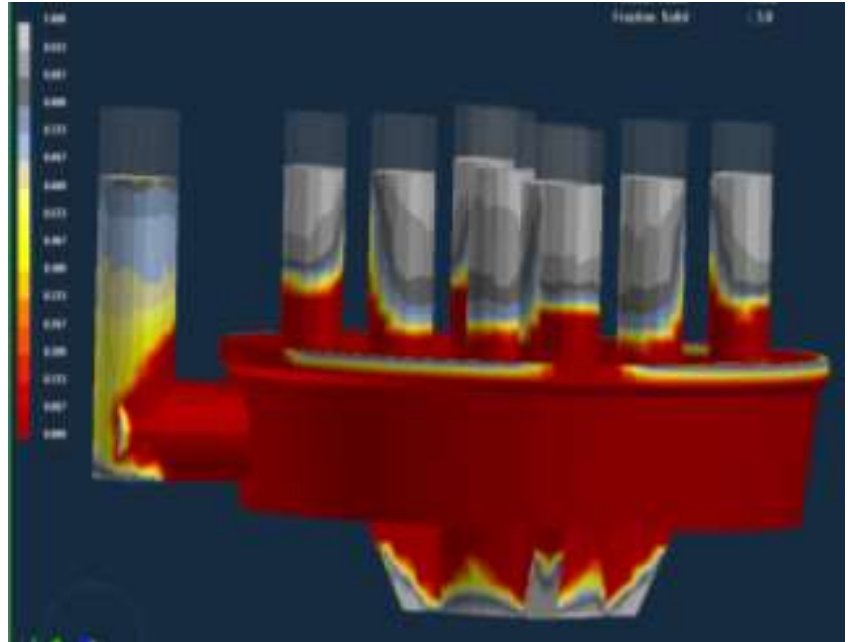

Figura 5. Solidificación de la pieza. Los colores rojo y gris indican temperaturas cercanas a $700^{\circ} \mathrm{C}$ y $300^{\circ}$, respectivamente.

La aleación A 380 tiene una composición química en peso en los rangos que muestra la tabla 1. Los datos usados en el programa jmat fueron los valores promedio se usaron los valores promedio de las concentraciones mostradas en la tabla mencionada.

La figura 6 muestra los valores del módulo de elasticidad o módulo de Young de la aleación A 380 en función de la temperatura. Es posible observar que el valor del módulo de Young a temperatura a $25^{\circ} \mathrm{C}$ es $\mathrm{E}=80 \mathrm{GPa}$, aproximadamente.

El valor del módulo incrementa rápidamente cuando la temperatura del material cambia de $650^{\circ} \mathrm{C}$ hasta $448^{\circ} \mathrm{C}$, debido a la transición en el cambio de fase líquida a sólida. Los valores todas las propiedades mecánicas cambian debido a la formación de fases microestructurales y la disminución del volumen especifico del material. Cabe mencionar que las propiedades de cualquier sistema simulado por medio de programas informáticos son una aproximación a las que pueden presentarse en forma experimental. Debido a las condiciones y variables de procesamiento que pueden variar durante el proceso de manufactura. Los valores teóricos o estimados en la simulación deberán ser comparados con valores reales obtenidos experimentalmente. 
Tabla 1. Composición química de la aleación A 380.

\begin{tabular}{cc}
\hline Elemento & \% en peso \\
\hline $\mathrm{Al}$ & Balance \\
$\mathrm{Cu}$ & $3.0-4.0$ \\
$\mathrm{Mg}$ & 0.1 \\
$\mathrm{Fe}$ & 1.3 \\
$\mathrm{Sn}$ & 0.35 \\
$\mathrm{Ni}$ & 0.5 \\
$\mathrm{Zn}$ & 3 \\
$\mathrm{Mn}$ & 0.5 \\
$\mathrm{Si}$ & $7.5-9.5$ \\
\hline
\end{tabular}

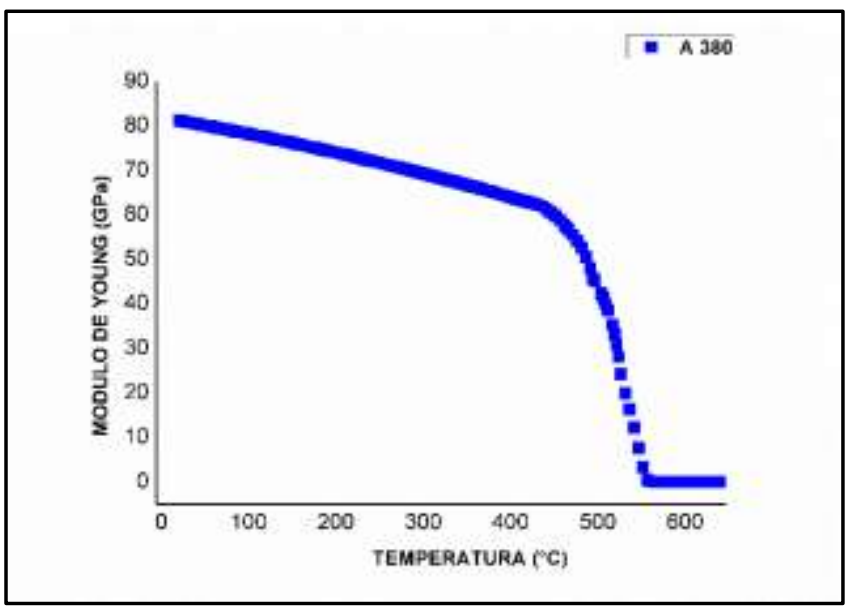

Figura 6. Módulo de elasticidad vs temperatura.

La figura 7 muestra el prototipo a escala 1:75 del modelo obtenido mediante impresión 3D. Es posible la obtención de modelos y piezas a partir de la aplicación de dicha técnica. Sin embargo, la calidad del modelo depende de las variables de impresión, mismas que dependen del tipo de filamento que se use en la construcción de piezas impresas. Entre los materiales que pueden serusados son ABS, PLA, fibra de carbono, entre otros. 4. El modelo a escala muestra que es posible la obtención de modelos, esto representa grandes ventajas respecto a la fabricación de modelos con el uso de madera y cera.

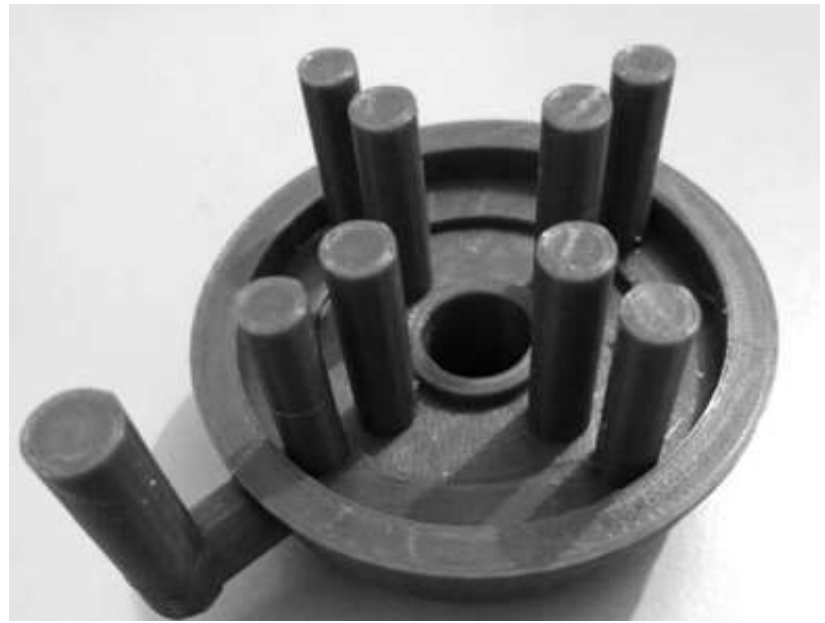

Figura 7. Pieza obtenida por impresión 3D en material PLA.

\section{Conclusiones}

La simulación del proceso de fundición en molde de arena fue posible con el uso de paquetes informáticos, al igual que la determinación del módulo de Young en función de la temperatura para la aleación A 380. Por medio de la impresión 3D se obtu vo un modelo a escala elaborado con un filamento de polímero PLA. Este trabajo resalta la importancia del uso de las tecnologías de la información para el diseño y desarrollo de piezas mecánicas. Sin embargo, es necesario comparar los valores de módulo de Young estimados o teóricos obtenidos por medio de la simulación respecto a los valores experimentales.

\section{Agradecimientos}

Los autores agradecen a SEP- PRODEP por el apoyo financiero del proyecto PRODEP-2019-0064 para la realización del presente trabajo de investigación. Finalmente, gracias a la Universidad Autónoma del Estado de Hidalgo, UAEH, por las gestiones otorgadas para la realización de este proyecto que involucra al personal académico y alumnos de la licenciatura en ingeniería mecánica.

\section{Referencias}

[1] Smith W. F. Ciencia e ingeniería de los materiales. McGraw-Hill; 2007.

[2] Groover M. Fundamentos de manufactura moderna. McGraw-Hill; 2007.

[3] https://www.dynacast.mx/a380 (enero 2020).

[4] https://impresiontresde.com/category/investiga cion/ (enero 2020). 